\title{
Zukunftschance Prozeßorientierung
}

\author{
Neben den bestehenden Gesetzen und Verordnungen sollen Unternehmen auch \\ Normen und Regelwerke in den Bereichen Umwelt, Qualiłäi, Arbeitsschutz und \\ Hygiene beachten. Dies ist mit erheblichem Aufwand verbunden. Ein Weg zur \\ Reduzierung dieses Aufwands liegt in der Integration der entsprechenden \\ Managementsysteme. Eine aktuelle Umfrage zeigt den derzeitigen Stand der \\ Praxis und beleuchtet Möglichkeiten sowie Probleme der Integration.
}

Von Stefan Enzler mmer mehr Regelungen beziehen sich inzwi_schen direkt auf Managementsysteme. Sehr deutlich zeichnet sich dieser Trend vor allem im Qualitätsmanagement und im Umweltmanagement ab (1). Im Arbeitsschutz- und Hygienemanagement verläuft die Entwicklung ähnlich (2). Unternehmen, die entsprechende Managementsysteme einrichten, müssen nicht nur die Vorgaben beachten, sondern die Systeme auch mit den eigentlichen Unternehmensabläufen koordinieren. Nur so sind die Unternehmen auf Dauer am Markt überlebensfähig.

Die Einfuihrung und Aufrechterhaltung der unterschiedlichen Managementsysteme bindet in den Unternehmen personelle und finanzielle Ressourcen in einer Weise, die insbesondere in kleinen und mittelständischen Unternehmen nicht mehr $\mathrm{zu}$ vertreten ist. Erschwerend kommt hinzu, daß die Managementsysteme häufig als schnelle Reaktion auf aktuelle Entwicklungen des Marktes implementiert werden und deshalb kaum miteinander verbunden sind. Eine Integration in die betrieblichen Prozesse und damit eine Unterstiit- zung der Wirtschaftlichkeit und der Effizienz des Unternehmens erfolgt in der Regel nicht, ebensowenig eine Optimierung und Verbesserung der Prozesse. Dies führt zu vergleichsweise hohem Aufwand bei geringem Nutzen.

\section{Steigender Integrationsbedarf}

Aufgrund dieser Problematik wächst in den Unternehmen das Bedürfnis nach einem Managementsystem, das die Anforderungen der Bereiche Umwelt, Qualität, Arbeitsschutz, Wirtschaftlichkeit und Hygiene in einem Gesamtzusammenhang berücksichtigt und in die bestehenden Abläufe und Prozesse integriert. Eine Studie des Instituts für Management und Umwelt von 1998 zum Integrierten Managementsystem, in der 500 Unternehmen der Lebensmittel-, Elektro- und Chemiebranche befragt wurden, belegt dies: 63,4 Prozent der befragten Unternehmen unterstreichen den Integrationsbedarf (3).

Die Studie zeigt, daß bereits 70 Prozent der befragten Unternehmen zwei Systeme eingeführt bzw. die Einführung geplant haben und sich somit mit der Integrationsproblematik auseinandersetzen müssen (vgl. Abb. 1). Insgesamt geben 42,2 Prozent an, daß sie bereits Teilsysteme integriert haben. Mehr als die Hälfte der befragten Unternehmen (54,4 Prozent) planen eine Integration der Systeme.

\section{Drei Ansätze in der Praxis}

In der betrieblichen Praxis sind unterschiedliche Ansät-
Abb. I: Implementierte und geplante Systeme fïr Qualitäi, Umwelischułz und Arbeitsschułz

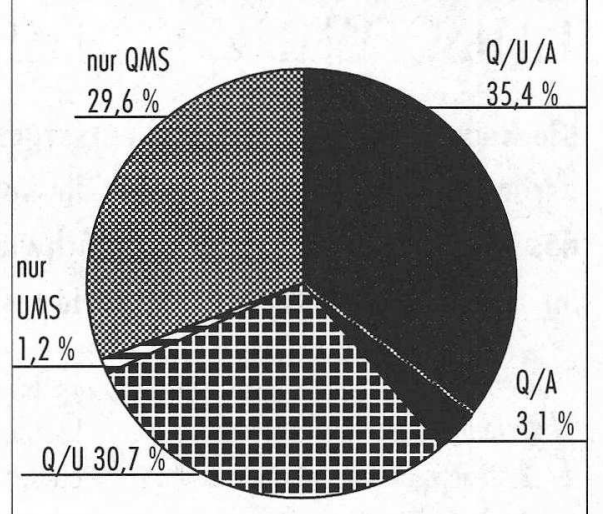

Quelle: eigene Darstellung

ze sogenannter „Integrierter Managementsysteme" anzutreffen, wobei sich drei Kategorien herausgebildet haben:

\section{Additiv integriert}

Die einzelnen Systeme stehen neben-einander und sind nur durch Querverweise in den unterschiedlichen Dokumentationen miteinander verbunden. Dieses Vorgehen, wie es zunächst Anfang der 90er Jahre durch das vermehrte Aufkommen von Umweltmanagementsystemen gebräuchlich wurde, ist ein Widerspruch in sich. Eine Addition der Systeme ist nicht mit einer Integration gleichzusetzen, sondern ist vielmehr als erster gedanklicher Einstieg in diese Thematik zu sehen.

\section{Partiell integriert}

Dieser Ansatz ist die Weiterentwicklung des additiven Ansatzes, indem zumindest einzelne Bereiche der Systeme zusammengeführt werden. Zumeist beschränkt sich dies auf eine Zusammenfuihrung der Dokumentation. Hierbei werden neu einzuführende Systeme in ein bereits bestehendes System eingefügt, z.B. Umweltmanagement und Arbeitsschutz als 21. und 22. Element des Qualitätsmanagements. Die Systemelemente bestehen so aber größtenteils völlig unabhängig voneinander weiter. Auch gegenwärtig wird dieser Ansatz, wie die Studie zeigt, trotz seines erhöhten Aufwandes noch zum überwiegenden Teil verwendet, da er von den Unternehmen auf den ersten Blick für die einfachste Vorgehensweise gehalten wird. Beispielsweise nutzen zwei Drittel der Unternehmen gemeinsame Verfahrens- und Arbeitsanweisungen sowie interne Audit-Verfahren. Gemeinsame Zertifizierungsverfahren führen hingegen nur 30 Prozent der Unternehmen durch. 


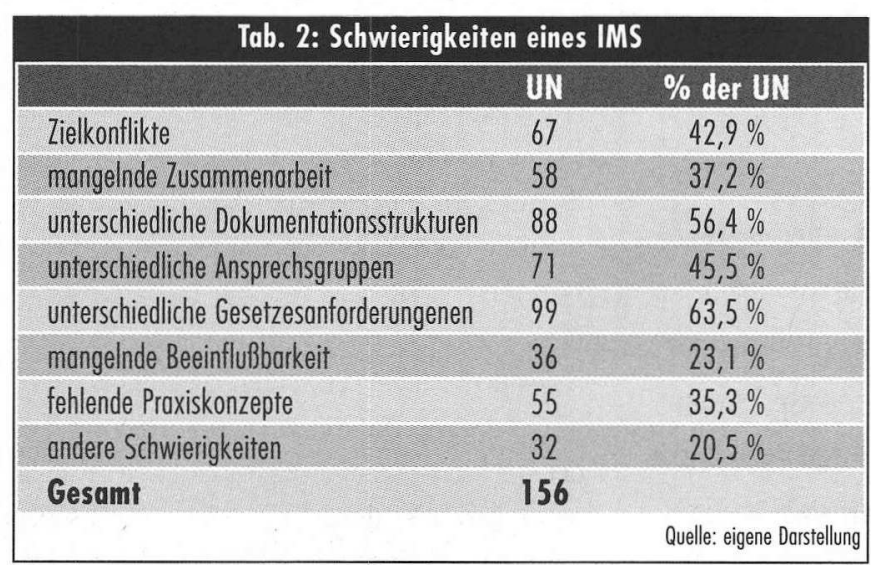

Die genannten Probleme weisen darauf hin, daß in der Unternehmenspraxis noch der Bedarf nach einer Unterstïtzung vorhanden ist. Sowohl bei der Homogenisierung der unterschiedlichen Gesetze, Normen und Verordnungen wie auch bei der Entwicklung von praxistauglichen Konzepten sind Staat und Wis-

\section{Systematisch integriert}

Hier existiert nur ein Managementsystem, das alle Anforderungen des Unternehmens erfiillt. Die gesamten Unternehmensanforderungen werden von der übergreifenden Steuerungsebene, dem eigentlichen Management, getrennt. Somit wird die integrierte Steuerung mehrerer ursprünglich verschiedener Systeme ermöglicht. Einen gewissen Bekanntheitsgrad auf diesem Gebiet hat das Integrierte Managementsystem von Hoechst (HIMS) erlangt (4). Der zukunftsträchtigste Ansatz ist hierbei die systemübergreifende Integration anband der betrieblichen Prozesse. In diesem Ansatz werden die Anforderungen der verschiedenen Managementbereiche nutzbringend und aufwandsreduzierend in die betrieblichen Prozesse integriert und durch ein übergreifendes Managementsystem gestaltet und gesteuert (5).

Als sinnvollstes Grundlagensystem wurde dabei von über 40 Prozent der Unternehmen der prozeßorientierte Ansatz für ein Integriertes Managementsystem hervorgehoben (vgl. Tab. 1).

Ausschlaggebend für die Integration von Managementsystemen ist bisher das Streben nach einer Reduktion des Aufwands. Diese Sichtweise unterstreicht die bisher noch weite Verbreitung des Ansatzes der partiellen Integration. Insbesondere genannt werden hier die Doppelarbeit bei Audits und die getrennte Dokumentation. Der Grund der Nutzenerhöhung durch die Optimierung der betrieblichen Prozesse wird hingegen kaum genannt.

\section{Schwierigkeiten der Integration}

Die bisherigen Erfahrungen mit der Einführung von Integrierten Managementsystemen zeigen auch unterschiedliche Schwierigkeiten auf. Ein Schwerpunkt liegt im Bereich der Dokumentationen, wie Tabelle 2 zeigt (Mehrfachantworten waren möglich). senschaft aktiv gefordert. Ob zur Lösung der Probleme die Einführung einer Norm für ein Integriertes Managementsystem hilfreich sein könnte, wurde von den befragten Unternehmen jedoch unterschiedlich beurteilt. Nur 10 Prozent halten eine solche Norm für notwendig, immerhin noch 43 Prozent für wünschenswert, während 46 Prozent sie als überflüssig einstufen.

\section{Anmerkungen}

(1) Für das Qualitätsmanagement ist hier vor allem das internationale Normsystem DIN EN ISO $9000 \mathrm{ff} z u$ nennen, aber auch Vorschläge des Total Quality Management

(TQM), der European Foundation for Quality Management (EFQM) und andere mehr. Für das Umweltmanagement sind insbesondere das EG-Öko-Audit-System (EMAS) und die internationale Norm DIN EN ISO 14001 von Relevanz.

(2) Vgl. z. B. Ritter, A./T. Langhoff: Arbeitsschutzmanagementsysteme - Vergleich ausgewöhlter Standards. 2. Auflage, Dortmund/Berlin 1998.

(3) Die Rücklaufquote von über 50 Prozent (257 Unternehmen) unterstreicht das Interesse der Unternehmenspraxis an der Entwicklung einer effizienten Lösung zur Integration von Managementsystemen.

(4) Vgl. Bock, H./ F. Riemenschneider: Das Hoechst Integrierte Management System, UmweltWirtschaftsForum 1/1997, S. 45-48.

(5) Vgl. z.B. Hessische Landesanstalt für Umwelt: Leiffaden Integrierte Managementsysteme, Wiesbaden 1997.

\section{Der Autor}

Stefan Enzler ist Gesellschafter am Institut für Management und Umwelt (imu).

Kontakt: imu, Gratzmüllerstraße 3, 86150 Augsburg, Tel. 0821/ 3490-271, Fax -273, E-mail: imu.enzler@a-city.de

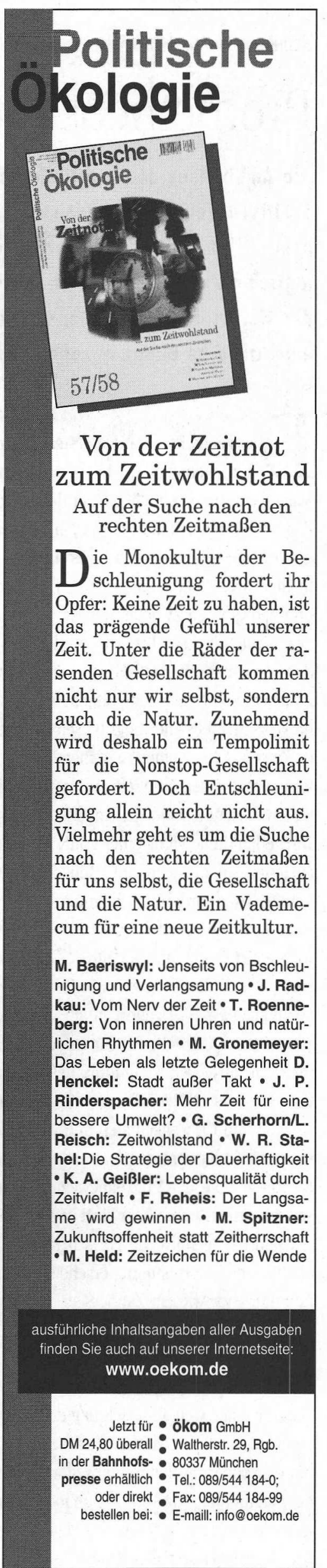


(c) 20I0 Authors; licensee IÖW and oekom verlag. This is an article distributed under the terms of the Creative Commons Attribution Non-Commercial No Derivates License (http://creativecommons.org/licenses/by-nc-nd/3.o/), which permits unrestricted use, distribution, and reproduction in any medium, provided the original work is properly cited. 